\title{
An Illumination Invariant Face Recognition Method Based on Gradientfaces and 2DLDA
}

\author{
Yongfeng Qi \\ College of Computer Science and Engineering, \\ Northwest Normal University, \\ Lanzhou Gansu 730070, PR China \\ tyyike@163.com
}

\author{
Yuanlian Huo \\ College of Physics and Electronic Engineering, \\ Northwest Normal University, \\ Lanzhou Gansu 730070, PR China \\ hylqqq@nwnu.edu.cn
}

\begin{abstract}
Extracting illumination invariant features is an effective method to improve recognition rate of algorithm with varying illumination. In this paper, a novel illumination invariant method which combines Gradientface and 2DPDA is proposed. First, this approach transforms a face image into Gradientface, then the 2DPCA is used to reduce the dimension of Gradientface. The experimental results verify the effectiveness of our approach on CAS-PEAR-R1 and CMU PIE face databases.
\end{abstract}

Keywords-Gradientfaces, 2DLDA, face recognition

\section{INTRODUCTION}

Due to the wide application prospects in pattern recognition fields, such as surveillance and security, face recognition has attracted the attention of many researchers in past decades. However, as mentioned in [1], "and "the variations between the images of the same face due to illumination are almost always larger than image variations due to change in face identity', varying illumination frequently makes recognition performance degradation.

Many methods have been proposed to handle the illumination variation problem. The methods includes: preprocessing and normalization ${ }^{[2][3][4]}, 3 \mathrm{D}$ illumination models ${ }^{[5][6]}$ and extraction of illumination invariant features ${ }^{[7][8]}$ In the first category, image processing techniques were used to preprocess face images. These techniques include histogram equalization, Gamma correction and logarithmic transformation. The main idea of the 3D illumination models is to represent the changes caused by different illuminations in a subspace and estimate model parameters. In the third category methods, facial features which are invariant or insensitive to illumination variations are extracted for recognition.

The gradient domain is very important to image processing. Zhang et al ${ }^{[9]}$ proposed Gradientface method for illumination invariance face recognition, in which the ratio of gradients at different directions ,denoted as Gradientface, were calculated and proved insensitive to the illumination changes. However, Gradientfaces contain some face-unrelated information and result in high dimensional problem. In this paper, we proposed a novel illumination invariant face recogn ${ }^{1}$ ition method, which

\footnotetext{
${ }^{1}$ The work is supported by the National Natural Science
} Foundation of China (No: 61262056) is a two stage method. In the first stage, Gradientfaces were used to reduce the impacts caused by changing illumination in face. And the second stage, $2 \mathrm{PCA}^{[10]}$ was used to extract the discriminant features for recognition.

\section{PROPOSED METHOD}

\section{A. Gradientface}

According to the physical imaging model, an image $I(x, y)$ is regarded as the product of the reflectance and illumination at each pixel $(x, y)$ :

$$
I(x, y)=R(x \cdot y)^{\top} L(x \quad y)
$$

where $R(x . y)$ is the reflectance and $L(x, y)$ is the illumination at each pixel $(x, y)$. The nature of $L(x, y)$ is determined by the illumination source, whereas $R(x \cdot y)$, which is regarded as an illumination-insensitive component, is determined by the characteristics of the image objects.

In order to separate these two independent components and to facilitate their separate processing, logarithm transformation is applied in Eq. (1), thus

$$
\tilde{I}(x, y)=\tilde{R}(x, y)+\tilde{L}(x, y)
$$

Where $\tilde{I}(x, y)=\ln (I(x, y)), \tilde{R}(x, y)=\ln (R(x, y))$ and $\tilde{L}(x, y)=\ln (L(x, y))$.

Considering two neighbor point $(x, y)$ and $(x+\triangle x, y)$ of an image $I(x, y)$ taken under lighting, since $L(x, y)$ is determined by the lighting source, then it is reasonable that $L(x, y)$ and $L(x+\triangle x, y)$ are approximately equal in general when $\Delta x$ is small. Therefore $L(x, y)$ is approximately smooth and $\partial \tilde{L}(\mathrm{x}, \mathrm{y}) / \partial \mathrm{x}=0$, $\partial \tilde{L}(x, y) / \partial y=0$. Thus,

$$
\begin{aligned}
& \frac{\partial \tilde{I}(x, y)}{\partial x}=\frac{1}{R(x, y)} \frac{\partial R(x, y)}{\partial x} \\
& \frac{\partial \tilde{I}(x, y)}{\partial y}=\frac{1}{R(x, y)} \frac{\partial R(x, y)}{\partial y}
\end{aligned}
$$

According to illumination model, $\mathrm{R}$ can be considered as an illumination insensitive measure. And the ratio of $y$-gradient of 
$I(x, y)$ to $\mathrm{x}$-gradient of $I(x, y)$ is also an illuminant insensitive measure. However, the ratio of y-gradient of image to $x$-gradient of image might be infinitude derived by zero value of x-gradient of image. To avoid this situation occurred, the Gradientface was defined:

Gradientface $=\arctan \left(\frac{\partial \tilde{I}(x, y)}{\partial y} / \frac{\partial \tilde{I}(x, y)}{\partial x}\right) \in[0,2 \pi]$

To compute the gradient stably and reduce the effects of noise, Gaussian filter is applied in the logarithmic field of face images. Fig. 1 shows two face images and their Gradientfaces:

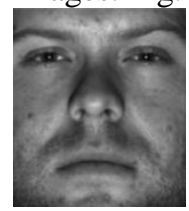

(a)

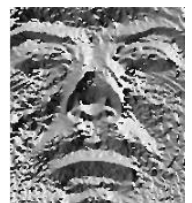

(b)

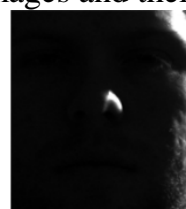

(c)

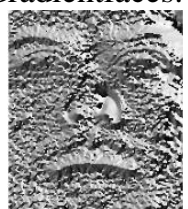

(d)
Fig.1 two face images and their Gradientfaces: (a) image1, (b) Gradientface of image1, (c) image2, (d) Gradientface of image2

\section{B. $2 D P C A$}

Let $Z \in R^{m \times d}$ be a matrix with orthonormal columns, $m \geq d$. Projecting the Gradientface $A_{i}$ onto $\mathrm{Z}$ yieldsThe total scatter of the projection feature matrices was used to determine $\mathrm{Z}$, namely

$$
\begin{aligned}
J(X) & =\operatorname{tr}\left\{E\left[(Y-E Y)(Y-E Y)^{T}\right]\right\} \\
& =\operatorname{tr}\left\{E\left[\left(Z^{T} A-E\left(Z^{T} A\right)\right)\left(Z^{T} A-E\left(Z^{T} A\right)\right)^{T}\right]\right\} \\
& =\operatorname{tr}\left\{Z^{T} E\left[(A-E A)(A-E A)^{T}\right] Z\right\}
\end{aligned}
$$

Define the image covariance matrix $G=E\left[(A-E A)(A-E A)^{T}\right]$, then $\mathrm{G}$ can be evaluated by

$$
G=\frac{1}{M} \sum_{i=1}^{M}\left(A_{i}-\bar{A}\right)\left(A_{i}-\bar{A}\right)^{T}
$$

Where T denotes matrix transpose, $\bar{A}$ is the average image as

$$
\bar{A}=\frac{1}{M} \sum_{i=1}^{M} A_{i}
$$

So

$$
J(Z)=Z^{T} G Z
$$

Thus, the optimal projection matrix $Z_{\text {opt }}=\left[z_{1}, \cdots, z_{d}\right]$ can be obtained by evaluating the eigenvectors corresponding to first d largest eigenvalues of $\mathrm{G}$.

\section{Feature extraction and classification}

Suppose that we have obtained the projection matrix $Z_{\text {opt }}$, projecting the Gradientface $A_{i}^{j}$ of each training image onto $Z_{\text {opt }}$ to yield its feature matrix

$$
Y_{i}^{j}=A_{i}^{j} X_{o p t}
$$

For a test Gradientface $A_{\text {test }}$, we have

$$
Y_{\text {test }}=A_{\text {test }} X_{\text {opt }}
$$

$Y_{\text {test }}$ is the feature matrix of test Gradient $A_{\text {test }}$.

The distance between feature matrix $Y_{\text {test }}$ and $Y_{i}^{j}$ is defined as:

$$
d\left(Y_{\text {test }}, Y_{i}^{j}\right)=\sum_{k=1}^{d}\left\|\left(Y_{\text {test }}\right)_{k}-\left(Y_{i}^{j}\right)_{k}\right\|_{2}
$$

Where $\left\|\left(Y_{\text {test }}\right)_{k}-\left(Y_{i}^{j}\right)_{k}\right\|_{2}$ denotes the Euclidean distance between vector $\left(Y_{\text {test }}\right)_{k}$ and vector $\left(Y_{i}^{j}\right)_{k}$. If $d\left(Y_{\text {test }}, Y_{l}^{k}\right)=\operatorname{Min} d\left(Y_{\text {test }}, Y_{i}^{j}\right)$ and $Y_{l}^{k}$ belongs to $l$-th class, then $A_{\text {test }}$ also belongs to $l$-th class.

\section{Experiment results}

In this section, experiments are designed to evaluate the performance of the proposed method. We compare our method with 2D-PCA and Gradientface. Experiments are conducted on two face databases, CAS-PEAL-R1 and CMU PIE.

\section{A. results on CAS-PEAL-R1 face database}

The CAS-PEAL-R1 face database ${ }^{[11]}$ contains30863 images of 1040 subjects (595 males and 445 females) with varying pose, expression, accessory, and lighting. In this experiment, lighting sub-databases was chosen for the evaluation of our proposed method. 199 persons are selected and each has 9 various illuminant pictures. We resize the images to $64 \times 64$ pixels with 256 gray levels.

We randomly choose 4 images per person to form training set and the other images for test. We average the result over 20 random splits. The top recognition results are shown in Table 1. From table 1 we can find the top recognition rate of the proposed method is higher than 2DPCA and Gradientface.

TABLE I. THE TOP RECOGNITION RATES OF THREE METHODS

\begin{tabular}{|c|c|c|c|}
\hline method & 2DPCA & $\begin{array}{c}\text { Proposed } \\
\text { method }\end{array}$ & Gradientface \\
\hline Recognition rate & 72.06 & 75.67 & 73.67 \\
\hline
\end{tabular}

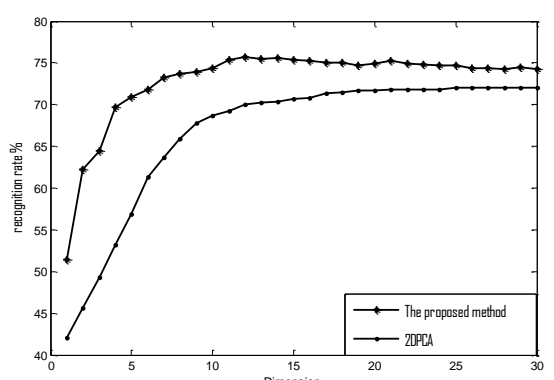

Fig.2 the recognition rate curves of 2DPCA and proposed method with various dimension of feature on CAS--PEAL-R1

To further compare the recognition performance of proposed method with 2DPCA. We also plot the recognition rates of the proposed method and 2DPCA with varying 
dimension of feature vectors in Fig.2 when the number of training samples is four. The Fig. 2 shows the proposed is of better recognition performance than 2DPCA under vary dimension

\section{B. results on CAS-PEAL-RI face database}

The CMU PIE ${ }^{[12]}$ database contains 41368 images of 68 people. Each person has images captured under 13 different poses and 43 different illumination conditions and with four different expressions. In this experiment, only frontal face images under different lighting conditions (without expression variations) are selected, and each person has 24 frontal images. Each image is manually aligned, cropped and resized to $32 \times 32$ pixels with 256 gray levels. Images of a person are showed in Fig.3.

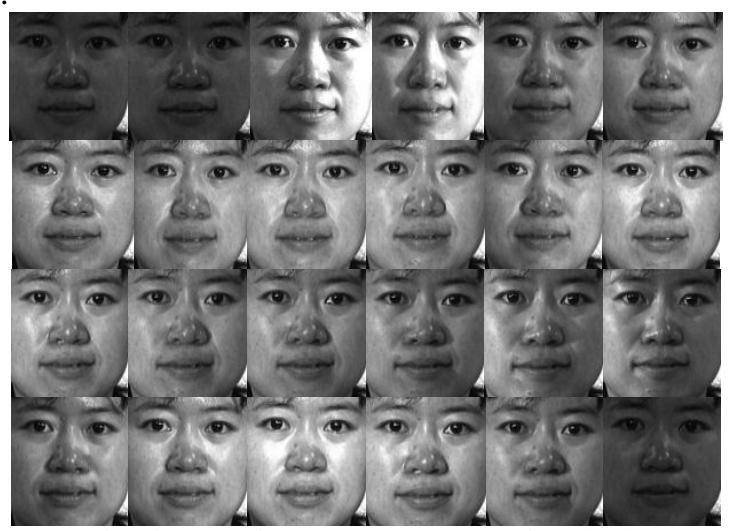

Fig. 3 images of a person on CMU PIE face database

In this experiment, we randomly choose 24 images per subject to form training set, and the other images for testing, we average the result over 20 random splits. The recognition results are shown in Fig.4. As can be seen, the proposed method has better recognition performance.

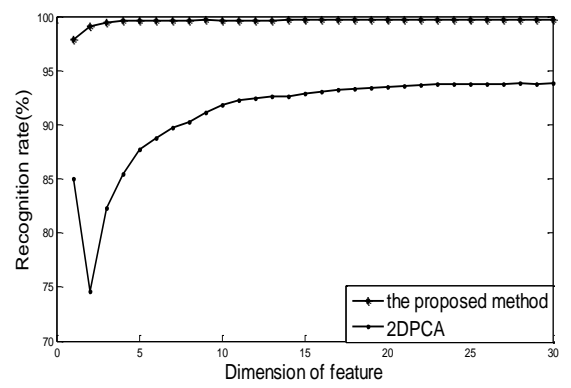

Fig.4 the recognition rate curves of 2DPCA and proposed method with various dimension of feature on CMU PIE

\section{Conclusions}

In this paper, a combining Gradientface and 2DPCA illumination invariant Face Recognition method is proposed. The method is a two-stage face recognition method. In the first stage, illumination invariant facial features are extracted by Gradientface. However, the feature dimensions extracted are very high, which will cost a lot of storage and computation capability. To improve the computational performance, the 2DPCA is applied to reduce the feature dimension.
Experimental results indicate that proposed method is of better recognition performance.

\section{ACKNOWLEDGMENT}

The authors gratefully acknowledge the support from the National Natural Science Foundation of China (No: 61262056).

\section{REFERENCES}

[1] Y.Adini, Y.Moses, S.Ullman, Face recognition: the problem of compensating for changes illumination direction, IEEE Transaction on Pattern Analysis and Machine Intelligence, 1997,19:721-732.

[2] Pizer, S. M., et al., Adaptive histogram equalization and its variations, Computer vision, graphics, and image processing , 1987,39(3):355-368.

[3] Shan, S., et al., Illumination normalization for robust face recognition against varying lighting conditions". In:Analysis and Modeling of Faces and Gestures, 2003. AMFG 2003. IEEE International Workshop on, 2003, 157-164.

[4] Savvides, M. and B. V. Kumar, Illumination normalization using logarithm transforms for face authentication. In: Audio-and VideoBased Biometric Person Authentication, 2003, 549-556.

[5] A. S. Georghiades, P. N. Belhumeur, D. W. Jacobs, From few to many: illumination cone models for face recognition under variable illumination and pose, IEEE Transaction on Pattern Analysis and Machine Intelligence, 2001,23:643-660.

[6] R. Basri, D. W. Jacobs, Lambertian reflectance and linear subspaces, IEEE Transaction on Pattern Analysis and Machine Intelligence, 2003,25:218-233

[7] Y. Wu, Yi. Jiang and Y. Zhou, et al. Generalized Weber-face for illumination-robust face recognition. Neurocomputing, 2014,136:262267

[8] B. Nithya, Y. Bhavani Sankari, K. Manikantan, et al. Discrete Orthonormal Stockwell Transform Based Feature Extraction for Pose Invariant Face Recognition. Procedia Computer Science, 2015,45: 290299

[9] T. Zhang, Y. Tang, and B. Fang, et al. Face recognition under varying illumination using gradientfaces. IEEE Transactions on Image Processing ,2009,18(11): 2599-2606.

[10] [10] J.Yang, D. Zhang, A.F. Frangi, et al. Two-dimensional PCA: a new approach to appearancebased face representation and recognition, IEEE Transactions on Pattern Analysis and Machine Intelligence. 2004,26 (1): 131-137.

[11] W. Gao, B. Cao and S Shan, et al. The CAS-PEAL Large-Scale Chinese Face Database and Baseline Evaluations, IEEE Transactions on Systems, Man and Cybernetics, Part A: Systems and Humans, 2007,38(1):149-161

[12] T. Sim, S. Bake, M. Bsat. The CMU pose, illumination, and expression database. IEEE Transactions on Pattern Analysis and Machine Intelligence 2003; 25(12):1615-1618. 